\title{
Registro recente de harpia, Harpia harpyja (Linnaeus) (Aves, Accipitridae), na Mata Atlântica da Reserva Natural Vale do Rio Doce, Linhares, Espírito Santo e implicações para a conservação regional da espécie
}

\author{
Ana C. Srbek-Araujo ${ }^{1} \&$ Adriano G. Chiarello ${ }^{1,2}$
}

\begin{abstract}
1 Programa de Pós-Graduação em Zoologia de Vertebrados, Pontifícia Universidade Católica de Minas Gerais. Avenida Dom José Gaspar 500, Coração Eucarístico, 30535-610 Belo Horizonte, M inas Gerais, Brasil.
\end{abstract}

${ }^{2}$ Autor correspondente. E-mail: bradypus@pucminas.br

\begin{abstract}
Recent record of harpy eagle, Harpia harpyja (Linnaeus) (Aves, Accipitridae), in Atlantic forest of Vale do Rio Doce Natural Reserve, Linhares, Espírito Santo, Brazil and implications for the regional conservation of the species. The present communication reports an observation occurred in August 2005 of the harpy eagle, Harpia harpyja (Linnaeus, 1758), in the Vale do Rio Doce Natural Reserve (VRDNR), located in northern Espírito Santo state, southeastern Brazil. The analysis of this and past records of the species in this reserve indicates the presence of a resident population in the region encompassed by the VRDNR and the Sooretama Biological Reserve. These two reserves total circa 46,250 ha of lowland Atlantic forest (Tabuleiros forest), which are mostly well preserved. Besides the great extension of forests, the rich and abundant mammal fauna present in these two reserves contribute to the regional permanence of the harpy eagle.

KEY WORDS. Birds of prey; conservation; forest fragmentation; Tabuleiro forest.
\end{abstract}

RESUMO. A presente comunicação reporta o registro de um macho adulto de harpia, Harpia harpyja (Linnaeus, 1758), na Reserva Natural da Vale do Rio Doce (RNVRD), região norte do Espírito Santo, em agosto de 2005. A análise deste e de registros históricos da espécie nesta reserva indica a presença de uma população residente na região compreendida pela RNVRD e pela Reserva Biológica de Sooretama. Estas duas reservas, contíguas entre si, compreendem cerca de 46250 ha de Floresta Atlântica de baixada (Mata de Tabuleiro), na sua maior parte bem conservada. Além da grande extensão de floresta, a rica e densa fauna de mamíferos presente nestas reservas contribuem para a permanência das harpias na região.

PALAVRAS-CHAVE. Aves de rapina; conservação; fragmentação florestal; Mata de Tabuleiro.

A harpia ou gavião-real, Harpia harpyja (Linnaeus, 1758), é considerada a maior ave de rapina das Américas, sendo uma das maiores aves viventes do mundo (SICK 1997). A espécie ocorre do México à Bolívia e Argentina, estando presente em grande parte do Brasil (Sick 1997). A harpia tem se tornado rara na porção sudeste/sul do país (Albuquerque 1995, Galettı et al. 1997), sendo mais facilmente encontrada na região Amazônica (Sıck 1997). De acordo com a Lista da Fauna Brasileira Ameaçada de Extinção, a espécie está inserida na categoria Quase Ameaçada em nível nacional (MACHADo et al. 2005). Entretanto, a situação da espécie na Mata Atlântica é muito mais grave, estando citada em listas vermelhas estaduais do sul e sudeste: "Provavelmente Extinta" no Rio Grande do Sul (MARQues et al. 2002) e em Minas Gerais (MACHADo et al. 1998); “Cri- ticamente em Perigo" no Paraná (MIKICH \& BérnILS 2004), São Paulo (Probio/SP 1998) e Espírito Santo (I PEMA 2004); e “Em Pe rigo" no Rio de Janeiro (Alves et al. 2000).

A presente comunicação reporta o registro visual de harpia na Reserva Natural Vale do Rio Doce(RNVRD), área de Mata Atlântica de Tabuleiro da região norte do Espírito Santo. O registro foi realizado em 24 de agosto de 2005 (12:37 h) a 250/300 m da Estrada Oiticica (estrada não pavimentada, localizada no interior da reserva). O espécime pousou em gal ho a aproximadamente $20 \mathrm{~m}$ de altura, eriçou as penas (corpo e topete) e permaneceu observando o ambiente durante cerca de sete minutos. Registros fotográficos obtidos na ocasião foram enviados a especialista (José Fernando Pacheco) que, de acordo com as características de plumagem do espécime, indicou tratar-se de um macho adulto. 
A RNVRD localiza-se a $30 \mathrm{~km}$ ao norte do Rio Doce, entre os municípios de Linhares e Jaguaré (1906'-1918'S e 3945'4019'W). A reserva está inserida em uma das áreas de extrema importância biológica para a conservação da biodiversidade da Mata Atlântica, estando localizada no Corredor Central da Mata Atlântica (Mınıstério do MeIo Ambiente 2000). Com aproximadamente 22000 ha de extensão, a RNVRD representa $25 \%$ da área de Mata Atlântica Primária remanescente do Espírito Santo e constitui a segunda maior reserva de Mata dos Tabuleiros ou Zona Tabular Costeira (Hiléia Baiana) do Estado. Associada à Reserva Biológica de Sooretama (REBIO Sooretama; 24.250 ha), constitui um bloco contínuo de mata que representa cerca de $50 \%$ da área de cobertura original remanescente do estado (OLIVER \& SANTOS 1991) e a maior área de preservação ao norte do Rio de Janeiro. A reserva apresenta rel evo relativamente plano, com uma seqüência de colinas tabulares, apresentando altitudes entre 28 e 65 m (Jesus \& Rolım 2005). De acordo com a classificação de Köppen, o clima na região é do tipo tropical quente e úmido (Awi), apresentando estação chuvosa no verão e seca no inverno (JEsus \& Rolım 2005). A temperatura média anual é de 23,3ㄷ, variando entre 14,8 e 34,2드 (média das mínimas e máximas, respectivamente), com uma precipitação pluviométrica média anual de $1202 \mathrm{~mm}$, caracterizada por uma forte variabilidade entre anos (JESUs \& Rolım 2005). A RNVRD está localizada nos domínios da Floresta Ombrófila Densa, segundo o Mapa de Ve getação do Brasil (IBGE 1993), sendo classificada como Floresta Estacional Perenifólia por Jesus \& RoLım (2005), que representa uma tipologia intermediária entre a primeira e a Floresta Estacional Semidecídua. A rede de drenagens local revela-se dendrítica/dicotômica, sendo formada por um conjunto de córregos tributários do Rio Barra Seca (JESUs \& Rolim 2005). A área do entorno da reserva está constituída principalmente por pastagens, sendo também encontradas áreas destinadas ao cultivo de mamão, café e eucalipto, entre outras culturas, apresentando um contorno não regular (JESUs \& RoLIm 2005).

A presença de harpia na RNVRD é conhecida desde a década de 1970 (GALETTI et al. 1997), incluindo a visualização de um ninho da espécie no interior da reserva em 1992 (РACHECO et al. 2003). Outros registros recentes da espécie na Mata Atlântica foram efetuados no sul da Bahia (Galetti et al. 1997, Silveira et al. 2005), Espírito Santo (Galetti et al. 1997, Pacheco et al. 2003), Rio de Janeiro (PAcheco et al. 2003), São Paulo (Galettı et al. 1997) e Santa Catarina (Albuquerque 1995) (Tab. I).

No Espírito Santo a harpia parece estar em vias de desaparecimento, especialmente na região serrana, ao sul do Rio Doce (Simon 2000). No município de Santa Teresa, onde são encontradas três unidades de conservação (Reserva Biológica Augusto Ruschi, Estação Biológica de Santa Lúcia e Parque Municipal Natural de São Lourenço), a espécie existiu no passado, constando em estudos realizados por Augusto Ruschi (Rusch 1977), com osúltimos registros em 1990, deacordo com PACHECO et al. (2003). Em estudo mais recente, WILLIS \& ONIKI (2002) consideraram a harpia como espécie ausente no município.
Segundo THIOLLAY (1989), a harpia necessita de grandes áreas de vida, sendo estimados aproximadamente $100 \mathrm{~km}^{2}$ por casal. Para GALETTI et al. (1997), a espécie pode ser considerada rara no estado de São Paulo, e provavelmente também em Santa Catarina e Paraná, devido à perda de hábitat e à baixa densidade local de presas. De acordo com os autores, as reservas do sudeste do Brasil apresentam baixas densidades de presas potenciais da espécie, estando a presença das harpias nestes locais associada a movimentos migratórios sazonais (GALETTI et al. 1997). Por outro lado, os indivíduos presentes no norte do Espírito Santo e sul da Bahia seriam representantes de uma população residente de harpias, tendo em vista que nestas regiões as presas potenciais da espécie revelam-se mais abundantes (GALetTI et al. 1997). De fato, em estudo realizado no norte do Espírito Santo, Chiarello (1999, 2000a) registrou altas densidades relativas para várias espécies de mamíferos na RNVRD, incluindo várias presas potenciais da harpia.

GalettI et al. (1997) destacam ainda que os registros recentes da espécie em São Paulo foram efetuados apenas durante o inverno (julho), sugerindo a ocorrência de movimentos migratórios de espécimes vindos provavelmente de Misiones, na Argentina. De forma semelhante, Albuquerque (1995) associa os registros de harpia em Santa Catarina ao final do inverno e à ocorrência de frentes frias durante o outono (conhecimento popular), embora não considere a existência de uma população migratória de harpia para a região. Destaca-se também que os registros da espécie no estado do Rio de Janeiro foram obtidos tanto no inverno (junho de 1997) quanto no verão (janeiro de 1980 e dezembro de 1984) (PACHECo et al. 2003), não indicando, até o momento, que a espécie esteja presente no estado apenas sazonalmente.

Os registros de harpia na RNVRD foram efetuados em fevereiro de 1985 por PACHECo et al. (2003); em agosto de 1992 por Galetti et al. (1997); em vários meses durante o segundo semestre do mesmo ano, em novembro de 2000 e em abril de 2002 por PACHECO et al. (2003); e em agosto de 2005 (presente comunicação); com um registro da espécie na REBIO Sooretama em maio 1996 (PACHeCo et al. 2003). Estes dados indicam a ausência de sazonalidade nos registros de harpia no bloco contínuo de mata Sooretama/Linhares, uma vez que os registros encontram-se distribuídos ao longo do ano (estações seca e chuvosa). Adicionalmente, ressalta-se que os dois registros re centes de ninho de harpia foram realizados ao norte do Rio Doce, na RNVRD (norte do Espírito Santo), durante o segundo semestre de 1992 (PACHECo et al. 2003) e na Serra das Lontras (sul da Bahia), em março de 2000 (Silveira et al. 2005).

De acordo com o exposto acima, é muito provável que a região de Linhares/Sooretama abrigue uma das últimas populações residentes da espécie em áreas de Mata Atlântica de baixada (Tabuleiros), visto que este tipo de mata foi um dos mais afetados pelo desmatamento, crescimento de áreas urbanas e atividades agropecuárias. Neste contexto, é plausível que indivíduos em dispersão, provenientes do norte do Espírito Santo, 
Tabela I. Registros recentes de Harpia harpyja para a porção centro-sul da Mata Atlântica (estados costeiros do sul e sudeste do Brasil, incluindo o sul da Bahia), de acordo com dados disponíveis na literatura científica.

\begin{tabular}{|c|c|c|}
\hline Ano & Localidade & Referência Bibliográfica * \\
\hline 1980 & Imediações da BR-116, encosta da Serra dos Órgãos, Rio de Janeiro & PACHECO et al. 2003 \\
\hline 1980 & Pilões, Santa Catarina & AlbuQUerQue 1995 \\
\hline 1984 & Região das Agulhas Negras, Parque Nacional do Itatiaia, Rio de Janeiro & PACHECO et al. 2003 \\
\hline 1985 & Reserva Natural Vale do Rio Doce, Linhares, Espírito Santo & PACHECO et al. 2003 \\
\hline 1986 & Pedro Canário (extremo norte do estado), Espírito Santo & PACHECO et al. 2003 \\
\hline 1989 & Parque Estadual da Serra do Tabuleiro, Caldas da Imperatriz, Santa Catarina & AlbuQuerque 1995 \\
\hline 1989 & Cananéia, São Paulo & GALETII et al. 1997 \\
\hline 1990 & Reserva Biológica Augusto Ruschi, Santa Teresa, Espírito Santo & PACHECO et al. 2003 \\
\hline 1990 & Ariri, São Paulo & GALETTI et al. 1997 \\
\hline 1991 & Estação Experimental Pau-Brasil, Porto Seguro, Bahia & GaLettI et al. 1997 \\
\hline 1991 & Cananéia, São Paulo & GALETII et al. 1997 \\
\hline 1992 & Reserva Natural Vale do Rio Doce, Linhares, Espírito Santo & GaLetTI et al. 1997 \\
\hline 1992 & Reserva Natural Vale do Rio Doce, Linhares, Espírito Santo & PACHECO et al. 2003 \\
\hline 1993 & Cananéia, São Paulo & GaLettI et al. 1997 \\
\hline 1996 & Reserva Biológica de Sooretama, Sooretama, Espírito Santo & PACHECO et al. 2003 \\
\hline 1997 & Paraty, Rio de Janeiro & PACHECO et al. 2003 \\
\hline 2000 & Serra das Lontras, Bahia & SILVErRA et al. 2005 \\
\hline 2000 & Reserva Natural Vale do Rio Doce, Linhares, Espírito Santo & PACHECO et al. 2003 \\
\hline 2000 & Serrinha do Alambari, contrafortes da Serra da Mantiqueira, Rio de Janeiro & PACHECO et al. 2003 \\
\hline 2002 & Reserva Natural Vale do Rio Doce, Linhares, Espírito Santo & PACHECO et al. 2003 \\
\hline 2005 & Reserva Natural Vale do Rio Doce, Linhares, Espírito Santo & Presente comunicação \\
\hline
\end{tabular}

* Registros baseados em comunicação pessoal cedida a pesquisadores foram referenciados de acordo com o autor da publicação consultada (responsabilidade pela comunicação científica do registro). PACHECO et al. (2003) agrupam registros próprios, comunicações pessoais e citações de outros autores.

possam atingir eventualmente o extremo sul da Bahia ou, alternativamente, a região serrana do Espírito Santo, onde razoável cobertura florestal ainda persiste (região de Santa Teresa e Santa M aria de Jetibá, especialmente). Entretanto, é pouco provável que a população residente em Linhares/Sooretama visite áreas tão dispersas, separadas por dezenas de quilômetros (entre Linhares e a região serrana) ou mesmo por centenas de quilômetros (entre Linhares e o sul da Bahia) durante atividades rotineiras de forrageio, sendo, portanto, de extrema importância a manutenção desta população para a conservação regional da espécie. Felizmente, o combate à caça ilegal, ao desmatamento e à ocorrência de incên dios têm sido significativamente intensificados na RNVRD e na REBIO Sooretama (R.M. JESUS, comunicação pessoal), representando um grande avanço em relação ao passado recente daquelas reservas (CHIARELLO 2000b). Destaca-se, entretanto, que outras medidas mais específicas, como a estimativa do tamanho populacional e o monitoramento de indivíduos residentes, gerando conhecimento sobre o tamanho da área de vida e da dieta da espécie na região, revelam-se ações recomendadas e necessárias para o melhor conhecimento do real status de conservação da harpia na Mata
Atlântica de tabuleiro do norte do Espírito Santo.

\section{AGRADECIMENTOS}

Os autores são gratos à Companhia Vale do Rio Doce/ Instituto Ambiental Vale do Rio Doce, especialmente a Renato Moraes de Jesus, pelo financiamento e apoio logístico, e a José Fernando Pacheco, pela identificação da espécie e determinação do sexo do indivíduo fotografado.

\section{REFERÊNCIAS BIBLIOGRÁFICAS}

Albuquerque, J.L.B. 1995. Observations of rare raptors in Southern Atlantic Rainforest of Brazil. Journal of Field Ornithology, Berlin, 66 (3): 363-369.

Alves, M.A.S.; J.F. Pacheco; L.A.P. Gonzaga; R.B. Cavalcanti; M.A. Raposo; C. Yamashita; N. C. Maciel \& M. Castanheira. 2000. Aves, p. 113-124. In: H.G.Bergallo; C.F.D. Rocha; M.A.S. Alves \& M. VAN SLUYS. (Eds). A fauna ameaçada de extinção do estado do Rio de Janeiro. Rio de Janeiro, Editora da UERJ, 166p.

Chiarello, A.G. 1999. Effects of fragmentation of the Atlantic forest on mammal communities in south-eastern Brazil. 
Biological Conservation, Kidlington, 89: 71-82.

Chiarello, A.G. 2000 a. Density and population size of mammals in remnants of Brazilian Atlantic forest. Conservation Biology, Boston, 14: 1649-1657.

Chiarello, A.G. 2000 b. Influência da caça ilegal sobre mamíferos e aves das matas de tabuleiro do norte do estado do Espírito Santo. Boletim do Museu de Biologia Professor Mello Leitão, Nova Série, Santa Teresa, 11/12: 229-247.

Galettı, M.; M.A.P. Martuscelli \& I. SimÃo. 1997. Records of Harpy and Crested Eagles in the Brazilian Atlantic forest. Bulletin of British Ornithologists' Club, London, 117 (1): 27-31.

IBGE. 1993. Mapa de vegetação do Brasil. Rio de Janeiro, Fundação Instituto Brasileiro de Geografia e Estatística, 1p.

IPEMA. 2004. Lista de espécies da flora e fauna ameaçadas de extinção no estado do Espírito Santo. Disponível na World Wide Web em: http://www.ipema-es.org.br [acesso em 16 de agosto de 2006].

Jesus, R.M. \& S.G. Rolım. 2005. Fitossociologia da Mata Atlântica de Tabuleiro. Boletim Técnico da Sociedade de Investigações Florestais, Viçosa, 19: 1-149.

Machado, A.B.M.; G.A.B. Fonseca; R.B. Machado; L.M.S. Aguiar \& L.V. LINS. 1998. Livro vermelho das espécies ameaçadas de extinção da fauna de Minas Gerais. Belo Horizonte, Fundação Biodiversitas, 605p.

Machado, A.B.M.; C.S. Martins \& G.M. Drummond. 2005. Lista da fauna brasileira ameaçada de extinção. Belo Horizonte, Fundação Biodiversitas, 160p.

Marques, A.A.B.; C.S. Fontana; E.Vélez; G.A. Bencke; M.Schneider $\&$ R. E.DOS REIS. 2002. Lista de referência da fauna ameaçada de extinção no Rio Grande do Sul. Porto Alegre, FZB/MCT, PUCRS/PANGEA, Publicações Avulsas FZB 11, 52p.

MIKICH, S.B. \& R.S. BÉRNILS. 2004. Livro vermelho da fauna ameaçada no estado do Paraná. Disponível na World Wide
Web em: http://www.pr.gov.br/iap [acesso em 09 de fevereiro de 2006].

MinistéRIO do MeIo Ambiente. 2000. Avaliação e ações prioritárias para a conservação da biodiversidade da Mata Atlântica e Campos Sulinos. Brasília, MMA/SBF, 40p.

OLIVER, W.L.R. \& I.B. SANTOS. 1991. Threatened and endemic mammals of the Atlantic Forest region of South-East Brazil. Wildlife Preservation Trust Special Science, Trinity, Jersey, 4: 1-26.

PACheCo, J.F.; P.S.M. Fonseca \& R.PARRINı. 2003. Coletânea cronológica de registros recentes de Harpia harpyja (L.) para os estados do Rio de Janeiro e Espírito Santo. Atualidades Ornitológicas, Londrina, 111: 7-10.

Probio/SP. 1998. Fauna ameaçada no estado de São Paulo. São Paulo, Secretaria de Estado do Meio Ambiente, Série Probio, SMA/CED, 56p.

Ruschl, A. 1977. A Ornitofauna da Estação Biológica do Museu Nacional. Boletim do Museu de Biologia Professor Mello Leitão, Santa Teresa, 88: 1-10.

Sıck, H. 1997. Ornitologia Brasileira. Rio deJaneiro, Nova Fronteira, 912p.

Silveira, L.F.; P.F. Develey; J.F. Pacheco \& B.M. Whitney. 2005. Avifauna of the Serra das Lontras-Javi montane complex, Bahia, Brazil. Cotinga, Bedfordshire, 24: 45-54.

Sımon, J.E. 2000. Composição da avifauna da Estação Biológica de Santa Lúcia, Santa Teresa - Espírito Santo. Boletim do Museu de Biologia Professor Mello Leitão, Nova Série, Santa Teresa, 11/12: 149-170.

THIOLLAY, J.M. 1989. Area requirements for the conservation of rain forest raptors and game birds in French Guiana. Conservation Biology, Boston, 3: 128-137.

Willis, E.O. \& Y.OnIKı. 2002. Birds of Santa Teresa, Espírito Santo, Brazil: do humans add or subtract species? Papéis Avulsos de Zoologia, São Paulo, 42 (9): 193-264.

Recebido em 31.III.2006; aceito em 20.X.2006. 